\title{
Analysis of Nonlinear Dynamics for Abrupt Change of Interphase Structure in Liquid-Liquid Mass Transfer
}

\author{
DongXiang Zhang, ${ }^{1}$ Min Sun, ${ }^{2}$ and Jing $\mathrm{Li}^{2}$ \\ ${ }^{1}$ School of Chemical Engineering and the Environment, Beijing Institute of Technology, \\ Beijing 100081, China \\ ${ }^{2}$ College of Applied Science, Beijing University of Technology, Beijing 100124, China
}

Correspondence should be addressed to Jing Li, leejing@bjut.edu.cn

Received 22 December 2008; Revised 30 April 2009; Accepted 4 May 2009

Recommended by Elbert E. Neher Macau

\begin{abstract}
As a liquid-liquid system is far from equilibrium state, the phase thickness is variable when mass transfer process with chemical reaction occurs in interphase zone, and a dispersible transitional layer called the interphase dispersed zone (IDZ) is formed. The IZD model composed of thermodynamically instable $\mathrm{O} / \mathrm{W}$ or $\mathrm{W} / \mathrm{O}$ microemulsion has reasonably explained enormous experimental phenomena in nonlinear mass transfer. To forecast the possible parameter ranges of IDZ process and abrupt change of liquid-liquid mass transfer rate, the dynamic characteristics of a molecular diffusion model are considered in this paper. We applied the bifurcation theory of planar dynamical system, Laplace transform, and maple software to investigate the model, and obtain different phase portraits of the system in different regions. The results obtained will play an important directive role in the study of IDZ model.
\end{abstract}

Copyright (c) 2009 DongXiang Zhang et al. This is an open access article distributed under the Creative Commons Attribution License, which permits unrestricted use, distribution, and reproduction in any medium, provided the original work is properly cited.

\section{Introduction}

Liquid-liquid mass transfer and reaction mass transfer processes are widely used in many industries such as nuclear energy, hydrometallurgy, pharmaceutical, and chemical. In research on production process control and techniques, we found that interphase processes are main control procedures for these liquid-liquid systems. In mass transfer process, the interphase zone always evolves with time, accompanying with chemical reaction, hyedromchanics instability, automatic dispersion, formation of interphase layer, absorption and coalescence of dispersed particles, and formation of interphase layer structure, etc. [1-3].

Traditional dynamic research methods for interphase mass transfer kinetics in liquidliquid systems use concentration difference to express the driving force for mass transfer, 
and assumed that each phase is unchangeable even in the interface and interphase layers have different thicknesses. However, such assumption is unable to explain the bifurcation of mass transfer rate in liquid-liquid systems under the effect of mechanic field, electric field, magnetic field, gravitational field, and sound field, etc. [1-7]. Abrupt change of mass transfer rate has great effect on theoretical research and production practice and becomes an important content of theoretical research and engineering security control. To overcome the limit in traditional understanding, Tarasov et al. have developed the physical model of IDZ through analyzing actual process phenomena[1-3]. Taking the mass transfer process in liquid-liquid stripping system as an example, Zhang holds that, due to the effect of coordinate chemical binding between extractant and extract as well as intermolecular function between coordination compound and solvent molecules, when the extracted ion carries the extractant and solvent particles dispersing near the inorganic interphase layer in the process of transferring from organic to inorganic phase, the extractant and the solvent after separating from the ion can form an $\mathrm{O} / \mathrm{W}$ microemulsion near the inorganic interphase zone under the competition of water molecules in the inorganic phase of other stripping agents. Similarly, the extract in the inorganic phase in liquid-liquid extraction system can also form a $\mathrm{W} / \mathrm{O}$ microemulion near the organic interphase layer. Under the effect of the thermal movement of molecule and concentration driving force, the two types of microemulsion come into being dynamically and will change dynamically during mass transfer process. Moreover, the $\mathrm{O} / \mathrm{W}$ microemulsion in the inorganic interphase and the $\mathrm{W} / \mathrm{O}$ microemulsion near the organic interphase layer can come into being simultaneously in the interphase layer of the mass transfer process. It is inferable that a bicontinuous-phase microemulsion can also be produced between the $\mathrm{O} / \mathrm{W}$ microemulsion and the $\mathrm{W} / \mathrm{O}$ microemulsion in the interphase layer [8]. This process is generally known as mass transfer microemulsion process in liquid-liquid system interface. This model for nonhomogenous mass transfer or reaction mass transfer process in the interface is called the model of IDZ[3].

In the reactor shown in Figure 1, we have obtained the molecular diffusion equation for interphase mass transfer $\partial c / \partial t=D\left(\partial^{2} c / \partial x^{2}\right)$ and the equation describing interphase mass transfer process $\partial c / \partial t=D\left(\partial^{2} c / \partial x^{2}\right)+r$ through equilibrium calculations by carrying out experiments for extraction and stripping of inorganic acid and transitional metal compounds. When a small-range of external electrostatic field was applied in the system, the bifurcation phenomenon was found in interphase mass transfer rate (refer to Figure 2).

When using any amine extractant to extract hydrochloric acid, there also appears a sudden change of the interphase mass transfer velocity when the initial concentration of hydrochloric acid is adjusted a little [3]. The phenomenon of the sudden change of the interphase mass transfer velocity caused by the microranged concentration adjustment cannot be explained with Fick's Law of diffusion. To coordinate the contradiction, Zhang and Trasov gave an explanation that along with the transfer of the key components in the phase interface areas, the water phase and organic phase form an IDZ structure, that is, water-in-oil emulsion, which gives rise to changes in interphase structure $[2,8]$.

Koltsova et al. have simplified the mass transfer interhpase layer into noncontinuous fluid film mode with interspace, and studied the IDZ process by using fractal dynamic model $\partial^{\alpha} c_{\alpha} / \partial t^{\alpha}=D_{\alpha}\left(\partial^{2} c_{\alpha} / \partial x^{2}\right)+k c_{\alpha}^{m}(0<\alpha<1)$ through simulation of the impetus of nonequilibrium thermodynamics [6,7]. Since the effect of chemical reaction rate $k c_{\alpha}^{m}$ in these systems is much less than that of diffusion, we have used $\partial^{\alpha} c_{\alpha} / \partial t^{\alpha}=D_{\alpha}\left(\partial^{2} c_{\alpha} / \partial x^{2}\right)$ for quantitative research on the change of interphase layer thickness and dispersive characteristics of medium induced by applying mechanical function in the interphase layer zone (refer to Figures 3 and 4) [6]. 


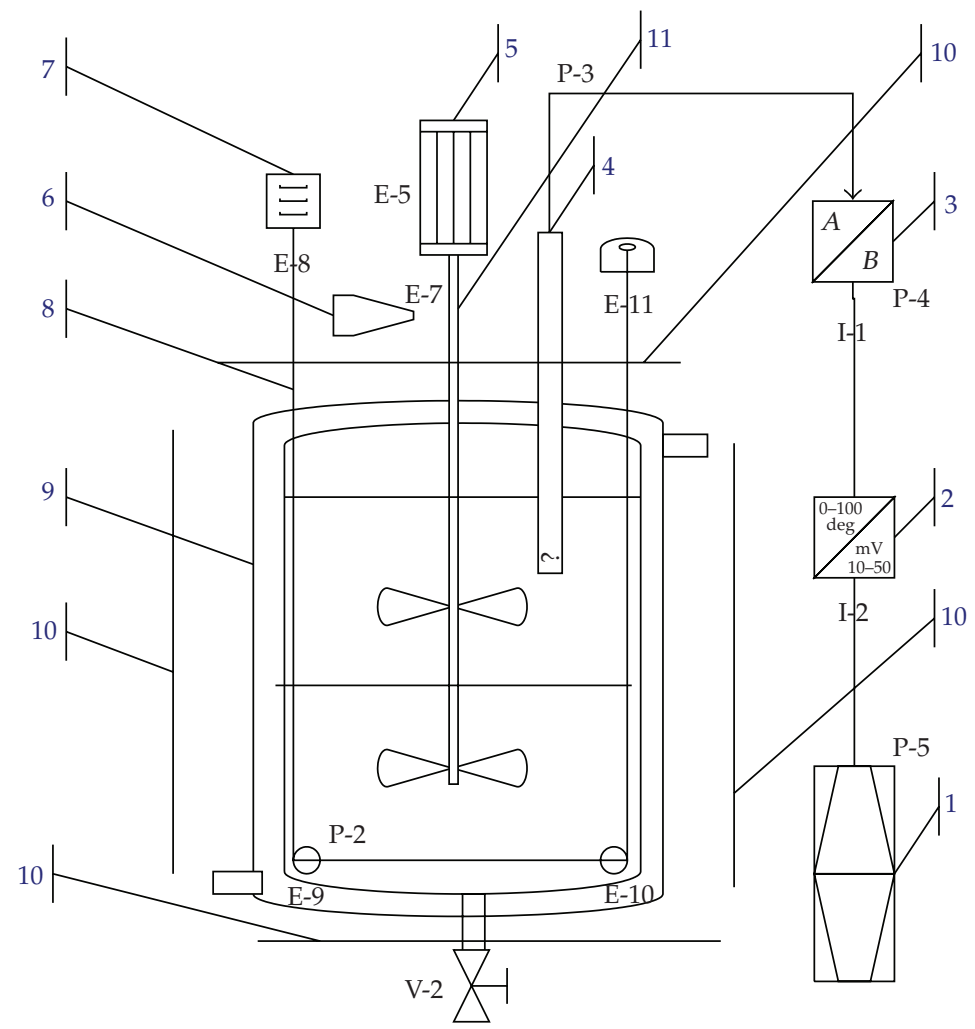

Figure 1: The improved constant interface cell 1-computer; 2-Digital switch-device; 3-concentration detector; 4-sensor; 5-constant speed motor; 6-speed device; 7-reciprocating device; 8-ribbon; 9-heat preserved water-jacket; 10-electric fields 11-stirrers.

Numerous researches show that the change of internal factors and the effect of external fields in mass transfer process can cause non-linear interphase molecular diffusion and abrupt change of interphase structure in liquid-liquid mass transfer. This also makes those parameter ranges in liquid-liquid mass transfer rate that may change abruptly a basic theoretical issue that has aroused common attention of researchers.

This paper studies the dynamic characteristics and phase diagram of molecular model $\partial c / \partial t=D\left(\partial^{2} c / \partial x^{2}\right)$ in liquid layer. The possibility of abrupt change of the reaction mass transfer process $\partial c / \partial t=D\left(\partial^{2} c / \partial x^{2}\right)+r$ is forecast through the basic mass transfer process $\partial c / \partial t=D\left(\partial^{2} c / \partial x^{2}\right)$ for liquid-liquid molecular diffusion.

The bifurcation theory of the plane polynomial vector fields plays an important role in the study of nonlinear dynamic system. By using the bifurcation theory, Li et al. [9] are considered a parametrically and externally excited mechanical system. Li et al. [10] investigated the rotor-AMB system with time-varying stiffness of single degree of freedom and found that there exist, respectively, at least 17, 19, 21, and 22 limit cycles in the system under four different control conditions. The dynamic characteristics of a molecular diffusion model are considered in this paper, we applied the bifurcation theory of planar dynamical system, laplace transform, and maple software to investigate the model, we obtain different phase portraits of the system in different regions. The results obtained will play an important directive role in the study of IDZ model. 


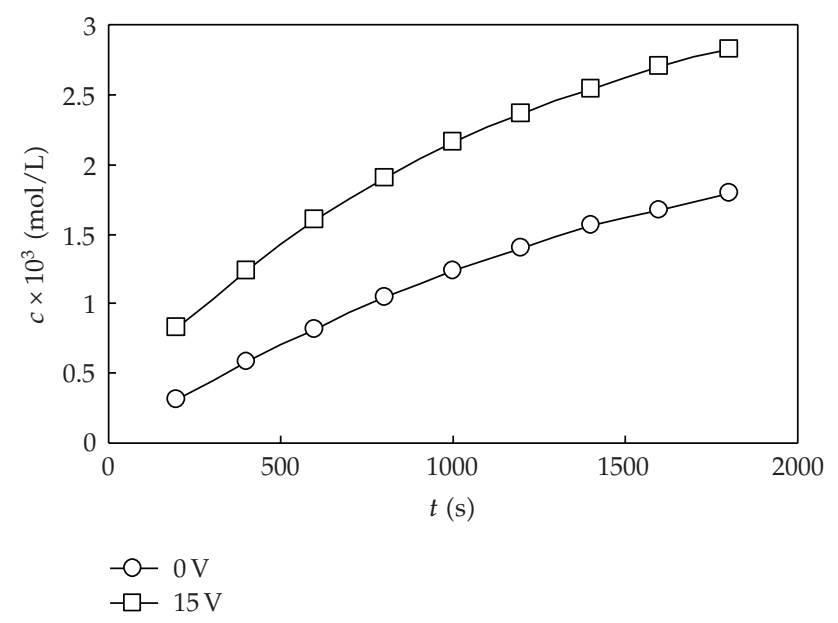

Figure 2: The changing of $\mathrm{Cu}^{2+}$ concentration in inorganic-phase along with the mass transfer process.

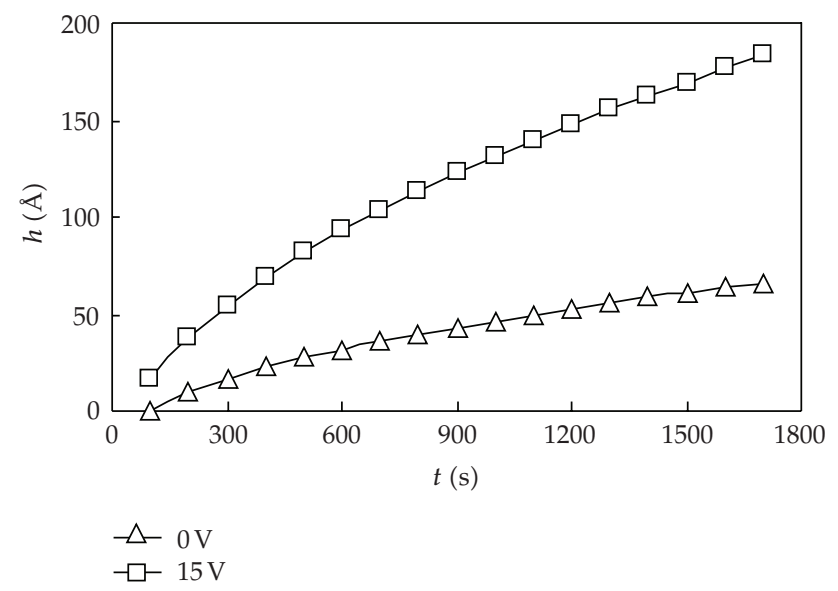

Figure 3: Curves of the interphase layer film thickness evolving with time contrasting with external electrostatic field.

\section{Laplace Transform and the Averaged Equation}

Based on the mathematical model of double film molecular diffusion, the one-dimensional second-order differential equation with thickness as $\mathrm{h}$ for controlling molecular diffusion in phase interface can be considered as $\partial c / \partial t=D\left(\partial^{2} c / \partial x^{2}\right)$, where $t$ refers to time, $c(t, x)$ refers to the concentration of the transferring composition at the time $t$ and position $x$, and $D$ refers to molecular diffusion coefficient. Unit of quantities in the diffusion coefficient is $\left[\mathrm{m}^{2} / \mathrm{s}\right]$. To find the molecular diffusion coefficient the formula in diluted solutions [11] can be used: $D=7.4 \times 10^{-12}\left((\beta M)^{1 / 2} T / \mu \mathcal{v}^{\cdot 3 / 5}\right)$, where $M$ refers to molecular weight of solvent, $T$ refers to temperature and its units is $\mathrm{K}, \mu$ refers to coefficient of dynamic viscosity and its units is $\mathrm{mPa} . \mathrm{s}$, and $\beta$ refers to coalescence parameter of solvent molecules. 


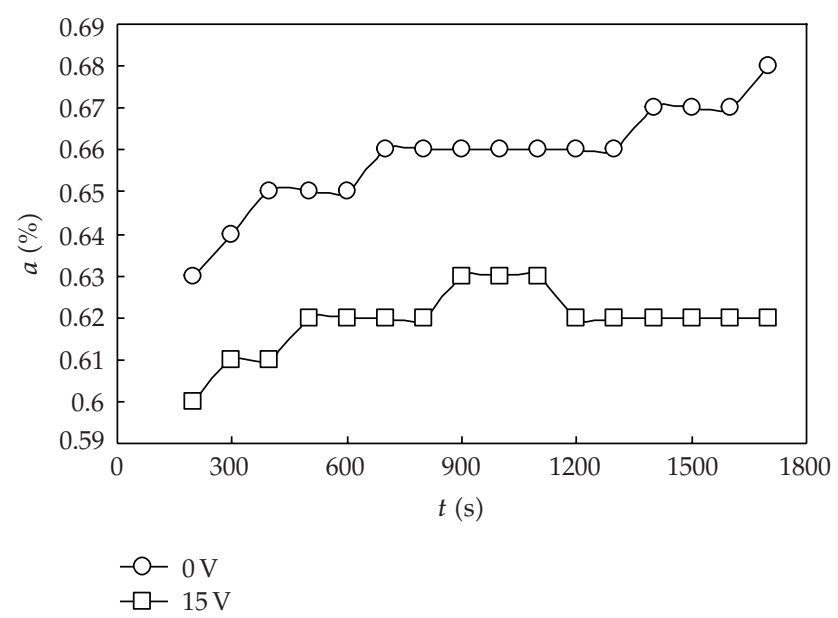

Figure 4: Changing curves of the interphase fractal dimension against time through comparing function of the external electrostatic field.

By using the Laplace transform to the following model as to $t$ :

$$
\frac{\partial c}{\partial t}=D \frac{\partial^{2} c}{\partial x^{2}}
$$

we get

$$
\bar{c}(p, x)=\int_{0}^{T} c(t, x) e^{-p t} d t
$$

From (2.2) we can obtain

$$
\bar{c}_{x x}=\int_{0}^{T} c_{x x} e^{-p t} d t .
$$

Simplify (2.2) we get

$$
\begin{aligned}
-p \bar{c}(p, x) & =\int_{0}^{T} c(t, x) d e^{-p t} \\
& =c(T, x) e^{-p t}-c(0, x)-\int_{0}^{T} e^{-p t} c_{t} d t
\end{aligned}
$$

Substitute $c_{t}=D c_{x x}$ to the above

$$
\begin{aligned}
-p \bar{c}(p, x) & =c(T, x) e^{-p t}-c(0, x)-\int_{0}^{T} D c_{x x} e^{-p t} d t \\
& =c(T, x) e^{-p t}-c(0, x)-D \bar{c}_{x x} .
\end{aligned}
$$


So

$$
\bar{c}_{x x}=\frac{c(T, x) e^{-p t}-c(0, x)+p \bar{c}(p, x)}{D} .
$$

Let

$$
f(\bar{c})=\frac{c(T, x) e^{-p t}-c(0, x)+p \bar{c}(p, x)}{D}=\frac{D_{T} x(\bar{c})}{D} .
$$

Using the Taylor series expansion to $f(x(\bar{c}))$ at the point $\bar{c}=0$

$$
f(x(\bar{c}))=f(x(0))+f^{\prime}(x(0)) \bar{c}+\frac{f^{\prime \prime}(x(0))}{2} \bar{c}^{2}+\cdots
$$

At the same time, we introduce Taylor series expansion to the right side of (2.7) at the point $\bar{c}=0$ :

$$
\frac{D_{T} x(\bar{c})}{D}=\frac{1}{D}\left(D_{T}(x(0))\right)+D_{T}^{\prime}(x(0)) \bar{c}+\frac{1}{2} D_{T}^{\prime \prime}\left(x(0) \bar{c}^{2}\right)+\cdots
$$

From (2.8) and (2.9), based on the two sides coefficient of $\bar{c}^{i}$ is equal, we can get

$$
\begin{aligned}
\bar{c}^{0}: f(x(0)) & =\frac{1}{D} D_{T}(x(0)), \\
\bar{c}^{1}: f^{\prime}(x(0)) & =\frac{1}{D} D_{T}^{\prime}(x(0)), \\
\bar{c}^{2}: f^{\prime \prime}(x(0)) & =\frac{1}{D} D_{T}^{\prime \prime}(x(0)), \\
& \vdots \\
\bar{c}^{i}: f^{(i)}(x(0)) & =\frac{1}{D} D_{T}^{(i)}(x(0)) .
\end{aligned}
$$

So,

$$
\begin{aligned}
\bar{c}_{x x} & =f(x(\bar{c})) \\
& =\frac{1}{D} D_{T}(x(0))+\frac{1}{D} D_{T}^{\prime}(x(0)) \bar{c}+\frac{1}{D} D_{T}^{\prime \prime}(x(0)) \bar{c}^{2}+\cdots+\frac{1}{D} D_{T}^{i}(x(0)) \bar{c}^{i}+\cdots
\end{aligned}
$$

Substitute $\bar{c}$ for $y$ and $x$ for $\tau$ for the convenience of our study, we get $y_{\tau \tau}=f(y)$. 
For any given initial values $D_{T}^{i}(x(0)), i \in N$, we can find the corresponding $f(y)$. Let $D_{T}^{i}(x(0))=0, i \in N$ and $i \neq 1,3,5, D_{T}^{\prime}(x(0))=D, D_{T}^{\prime \prime \prime}(x(0))=-a D$ and $D_{T}^{5}(x(0))=D / 16$, we can obtain $f(y)=y-a y^{3}+(1 / 16) y^{5}$.

Let $d y / d \tau=x+b x^{3}$, so $d x / d \tau=y_{\tau \tau} /\left(1+3 b x^{2}\right)=f(y) /\left(1+3 b x^{2}\right)$.

If we introduce the nonlinear transform $d \tau / d \xi=-\left(1+3 b x^{2}\right)$, then

$$
\begin{gathered}
\frac{d x}{d \xi}=\frac{d x}{d \tau} \frac{d \tau}{d \xi}=f(y) \\
\frac{d y}{d \xi}=\frac{d y}{d \tau} \frac{d \tau}{d \xi}=-x-4 b x^{3}-3 b^{2} x^{5}
\end{gathered}
$$

By means of the nonlinear transform, (2.6) is equivalent to the averaged equation

$$
\begin{gathered}
\frac{d x}{d \xi}=y-a y^{3}+\frac{1}{16} y^{5} \\
\frac{d y}{d \xi}=-x-4 b x^{3}-3 b^{2} x^{5}
\end{gathered}
$$

where $a$ and $b$ are parameters, $a>1 / 2, b<0$.

The Hamiltonian function of system (2.13) is

$$
H(x, y)=\frac{y^{2}}{2}-\frac{a y^{4}}{4}+\frac{y^{6}}{96}+\frac{x^{2}}{2}+b x^{4}+\frac{b^{2}}{2} x^{6}
$$

\section{Characteristic of the Singular Points}

The singular points of system (2.13) are

$$
\begin{gathered}
A_{00}(0,0), \quad A_{01}\left(0, \pm y_{1}\right), \quad A_{02}\left(0, \pm y_{2}\right), \quad A_{10}\left( \pm x_{1}, 0\right), \quad A_{11}\left( \pm x_{1}, \pm y_{1}\right) \\
A_{12}\left( \pm x_{1}, \pm y_{2}\right), \quad A_{20}\left( \pm x_{2}, 0\right), \quad A_{21}\left( \pm x_{2}, \pm y_{1}\right), \quad A_{22}\left( \pm x_{2}, \pm y_{2}\right)
\end{gathered}
$$

where $x_{1}=\sqrt{-1 / b}, x_{2}=\sqrt{-1 / 3 b}, y_{1}=2 \sqrt{2 a+\sqrt{4 a^{2}-1}}, y_{2}=2 \sqrt{2 a-\sqrt{4 a^{2}-1}}$.

For a singular point of system (2.13), its characteristic is determined by the eigenvalue of the polynomial

$$
p(\lambda)=\lambda^{2}-G(x, y)
$$

where

$$
G(x, y)=\left(1-3 a y^{2}+\frac{5}{16} y^{4}\right)\left(-1-12 b x^{2}-15 b^{2} x^{4}\right)
$$


By the bifurcation theory of the plane polynomial vector fields, for a singular point $\left(x_{i}, y_{j}\right)$ of a planar Hamiltonian system, if $G\left(x_{i}, y_{j}\right)>0$, then this singular point is a saddle point; if $G\left(x_{i}, y_{j}\right)<0$, then it is a center point; if $G\left(x_{i}, y_{j}\right)=0$, the singular point is a cusp [12].

Based on the bifurcation theory of the plane polynomial vector fields, it is found that the singular points $A_{00}(0,0), A_{01}\left(0, \pm y_{1}\right), A_{10}\left( \pm x_{1}, 0\right), A_{11}\left( \pm x_{1}, \pm y_{1}\right), A_{22}\left( \pm x_{2}, \pm y_{2}\right)$ are the centers, and the singular points $A_{02}\left(0, \pm y_{2}\right), A_{12}\left( \pm x_{1}, \pm y_{2}\right), A_{20}\left( \pm x_{2}, 0\right), A_{21}\left( \pm x_{2}, \pm y_{1}\right)$ are the saddle points.

From (2.14), we can obtain the value of the Hamiltonian function at the singular point of system (2.13), respectively,

$$
\begin{aligned}
& h_{0}^{c}=H\left( \pm x_{1}, \pm y_{1}\right)=s+t, \quad h_{1}^{c}=H\left( \pm x_{1}, 0\right)=0, \quad h_{2}^{s}=H\left( \pm x_{1}, \pm y_{2}\right)=s-t, \\
& h_{3}^{s}=H\left( \pm x_{2}, \pm y_{1}\right)=s+t-\frac{2}{27 b^{\prime}}, \quad h_{4}^{s}=H\left( \pm x_{2}, 0\right)=-\frac{2}{27 b^{\prime}} \\
& h_{5}^{s}=H\left( \pm x_{2}, \pm y_{2}\right)=s-t-\frac{2}{27 b^{\prime}}, \quad h_{6}^{c}=H(0,0)=0, \\
& h_{7}^{c}=H\left(0, \pm y_{1}\right)=s+t, \quad h_{8}^{s}=H\left(0, \pm y_{2}\right)=s-t,
\end{aligned}
$$

where $s=4 a-\left(32 a^{3} / 3\right), t=\left(4-16 a^{2}\right) \sqrt{4 a^{2}-1} / 3$.

\section{Bifurcation Parameter and Dynamic Analysis}

In order to compare $h_{2}^{s}, h_{3}^{s}, h_{4}^{s}$, we consider these curves:

$$
\left(C_{1}\right): h_{2}^{s}=h_{3}^{s}, \quad\left(C_{2}\right): h_{2}^{s}=h_{4}^{s}, \quad\left(C_{3}\right): h_{3}^{s}=h_{4}^{s} .
$$

These three curves and $a=1 / 2$ divided the plane into some different bifurcation regions as shown in Figure 5.

Where the region I is $1 / 2<a<\sqrt{3} / 3$ and $2 / 27(t-s)<b<0$, the region II is $a>\sqrt{3} / 3$, and $1 / 27 t<b<0$. The region III is $a>\sqrt{3} / 3$ and $2 / 27(t-s)<b<1 / 27 t$.

\section{Case I}

We consider the parameter condition of $U P=(a, b)=(0.6,-0.05) \in I I$, the phase portrait of system (2.13) as shown in Figure 6.

So, $h_{0}^{c}=-0.293150, h_{1}^{c}=0, h_{2}^{s}=0.485150, h_{3}^{s}=1.188330, h_{4}^{s}=1.481481, h_{5}^{c}=1.966632$, $h_{6}^{c}=0, h_{7}^{c}=-0.293150, h_{8}^{s}=0.485150$. We can obtain $h_{0}^{c}<h_{1}^{c}<h_{2}^{s}<h_{3}^{s}<h_{4}^{s}<h_{5}^{c}, h_{0}^{c}=h_{7}^{c}$, $h_{1}^{c}=h_{6}^{c}, h_{2}^{s}=h_{8}^{s}$.

Figure 7 demonstrates the changing process of the phase portraits as $h$ varied under the condition UP.

There are nine different families $\left\{\Gamma_{j}^{h}\right\},(j=1,2, \ldots, 9)$ of closed orbits for system (2.13) as the variable $h$ changes, which are described as follows: $\left\{T_{1 i}^{h}\right\}, h \in\left(h_{0}^{c}, h_{2}^{s}\right)$, four families closed orbits enclosing the singular points $\left( \pm x_{1}, \pm y_{1}\right) ;\left\{T_{2 i}^{h}\right\}, h \in\left(h_{0}^{c}, h_{2}^{s}\right)$ : two families closed orbits enclosing the singular points $\left(0, \pm y_{1}\right) ;\left\{T_{3 i}^{h}\right\}, h \in\left(h_{1}^{c}, h_{2}^{s}\right)$ : two families closed orbits enclosing the singular points $\left( \pm x_{1}, \pm y_{1}\right) ;\left\{T_{4}^{h}\right\}, h \in\left(h_{1}^{c}, h_{2}^{s}\right)$ : one family closed orbit 


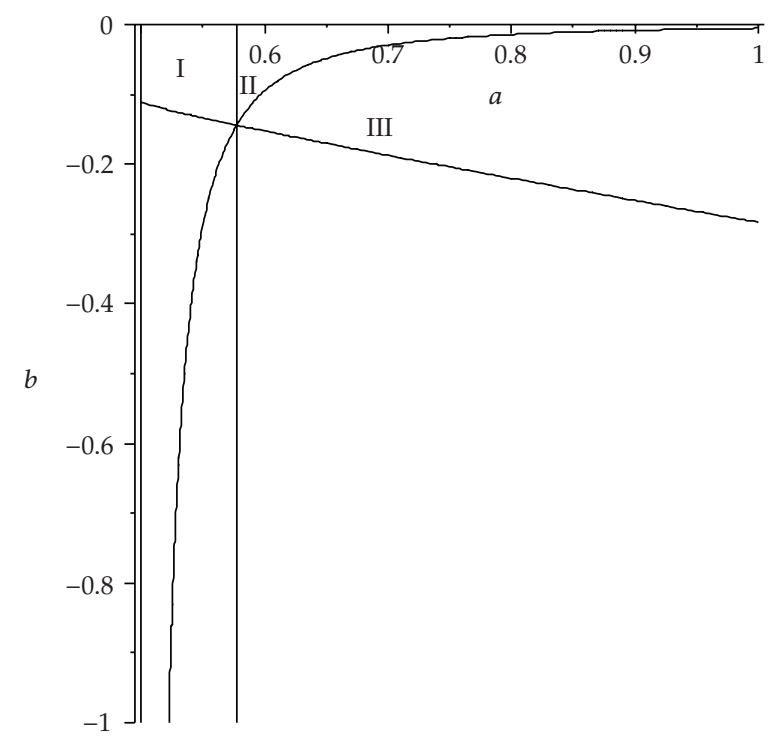

Figure 5: The bifurcation sets of the system (2.13).

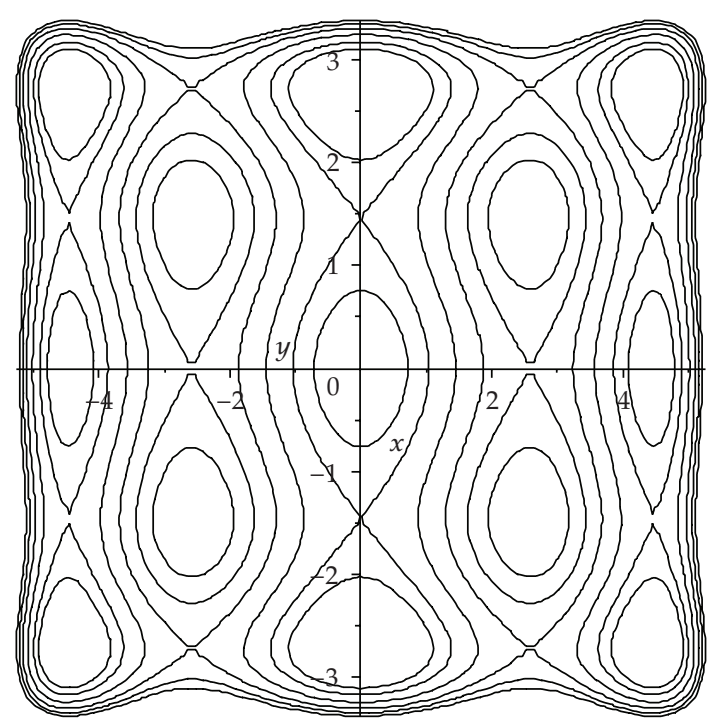

Figure 6: The phase portrait of system (2.13) under the condition UP.

enclosing the singular point $(0,0) ;\left\{T_{5 i}^{h}\right\}, h \in\left(h_{2}^{s}, h_{3}^{s}\right)$ : two families closed orbits enclosing the singular points $\left( \pm x_{1}, \pm y_{1}\right),\left( \pm x_{1}, \pm y_{2}\right)$ and $\left( \pm x_{1}, 0\right) ;\left\{T_{6}^{h}\right\}, h \in\left(h_{2}^{s}, h_{3}^{s}\right)$ : one family closed orbit enclosing the singular points $(0,0),\left(0, \pm y_{1}\right)$ and $\left(0, \pm y_{2}\right) ;\left\{T_{7 i}^{h}\right\}, h \in\left(h_{3}^{s}, h_{4}^{s}\right)$ : two families closed orbits enclosing the singular points $\left( \pm x_{2}, \pm y_{2}\right),\left( \pm x_{2}, 0\right) ;\left\{T_{8 i}^{h}\right\}, h \in\left(h_{4}^{s}, h_{5}^{c}\right)$ : four families closed orbits enclosing the singular points $\left( \pm x_{2}, \pm y_{2}\right) ;\left\{T_{9}^{h}\right\}, h \in\left(h_{3}^{s}, \infty\right)$ : the global closed orbit enclosing all the singular points.

Notice that as $h$ increases, the periodic orbits $T_{7}$ and $T_{8}$ contract inwards all other periodic orbits expand outwards. 


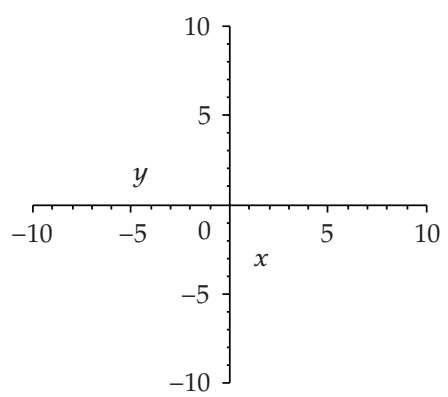

(a) $h=h_{0}^{c}$

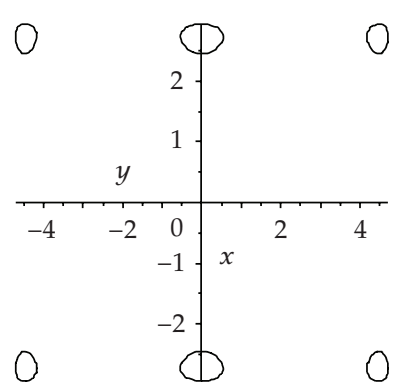

(b) $h_{0}^{c}<h<h_{1}^{c}$

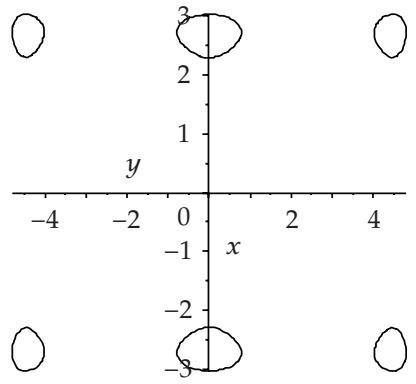

(c) $h=h_{1}^{c}$

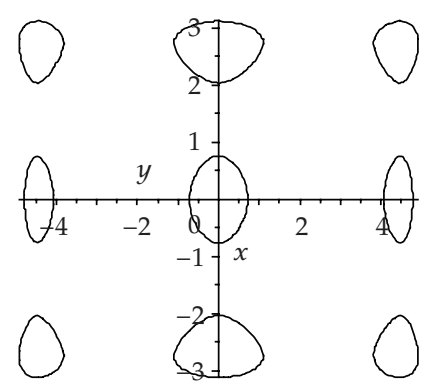

(d) $h_{1}^{c}<h<h_{2}^{s}$

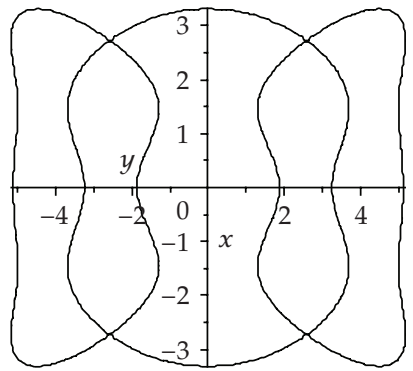

(g) $h=h_{3}^{s}$

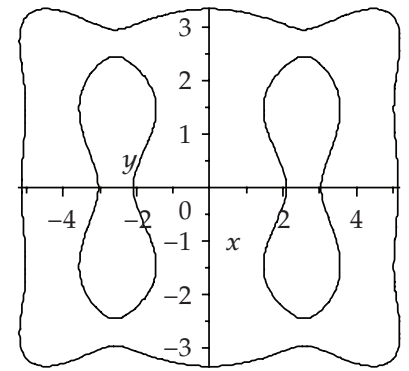

(h) $h_{3}^{s}<h<h_{4}^{s}$

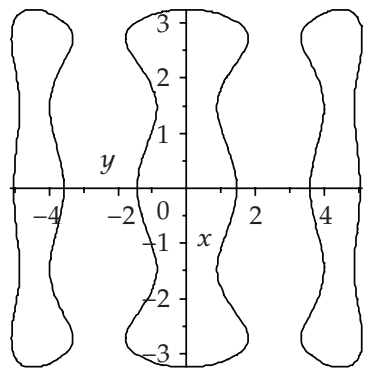

(f) $h_{2}^{s}<h<h_{3}^{s}$

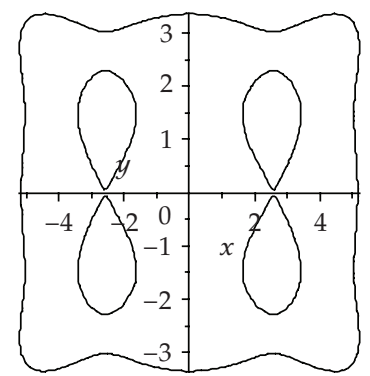

(i) $h=h_{4}^{s}$

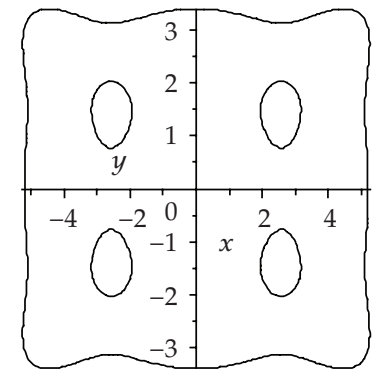

(j) $h_{4}^{s}<h<h_{5}^{c}$

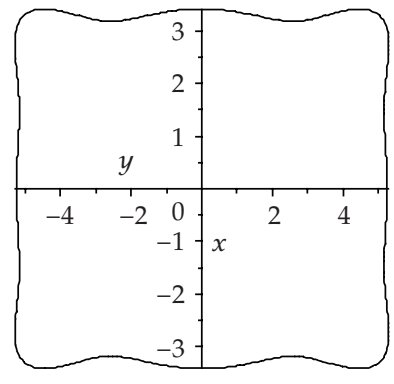

(k) $h=h_{5}^{c}$

Figure 7: The changing process of the phase portraits as $h$ varied under the condition UP. 


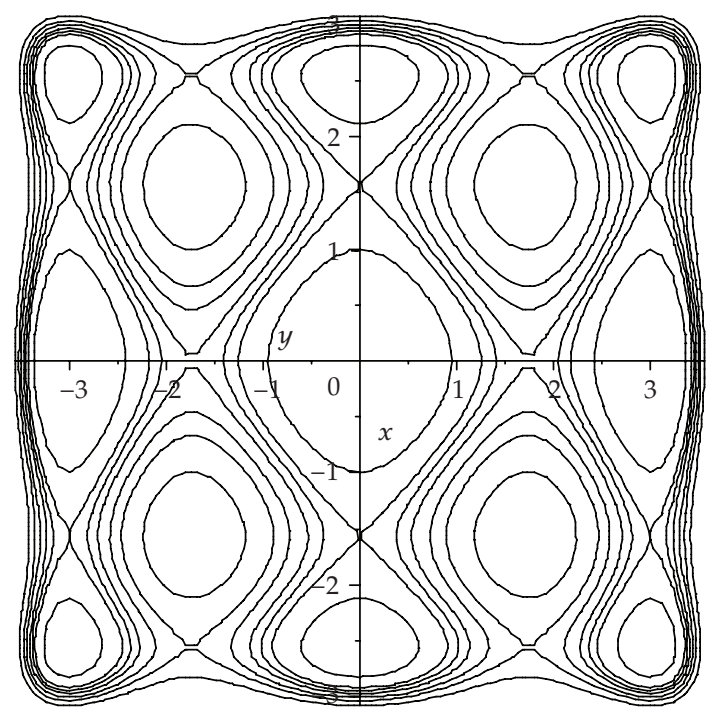

Figure 8: Phase portraits of system (2.13) in the region I.

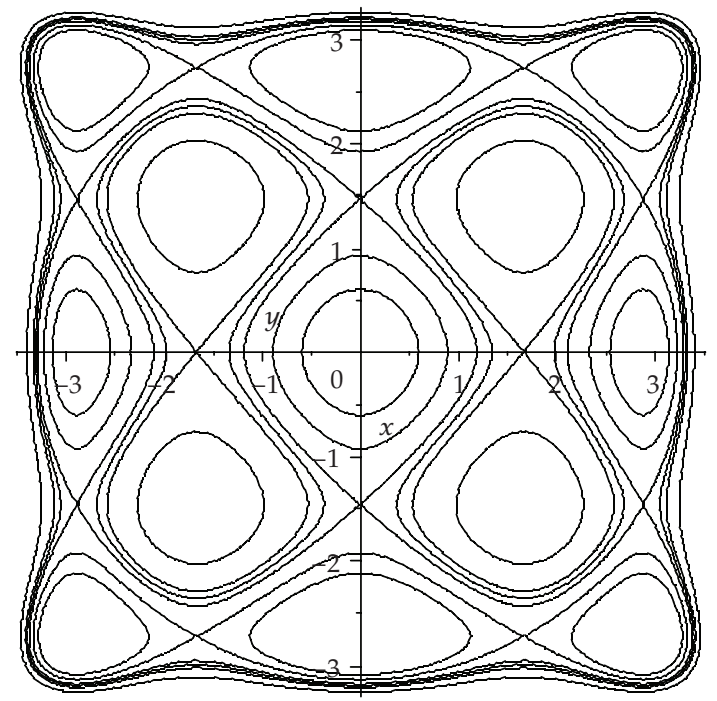

Figure 9: Phase portraits of system (2.13) in the region III.

The phase portraits are topologically equivalent to the Figure 6 when the parameters $(a, b) \in I I$.

\section{Case II}

When $a$ through the line $a=\sqrt{3} / 3$ from right to left that means the region II changes into the region I, the bifurcation happened. Figure 8 show the phase portraits of system (2.13) in the region $I$. 
The phase portraits are topologically equivalent to Figure 8 when the parameters $(a, b) \in I$.

\section{Case III}

When $b$ through the curven $b=1 / 27 t, t=\left(4-16 a^{2}\right) \sqrt{4 a^{2}-1} / 3$ from up to down that means the region II changes into the region III, the bifurcation happened. Figure 9 shows the phase portraits of system (2.13) in the region III.

The phase portraits are topologically equivalent to Figure 9 when the parameters $(a, b) \in I I I$.

Based on the above analysis, we know that the phase portraits are different from each other because there are in different regions.

\section{Conclusions}

The dynamic characteristics of a molecular diffusion model are considered in this paper, by using the bifurcation theory of planar dynamical system, Laplace transform, and maple software. First the model equation was transformed to the averaged equation by means of Laplace transform, and we obtain that when bifurcation parameters $(a, b)$ through the line $a=\sqrt{3} / 3$ and the curve $b=1 / 27 t$ the interphase structure will change suddenly, and then we get the phase portraits of system (2.13) in different regions. The results obtained enriches the dynamic research contents of the Liquid-liquid system, and have important theory values and apply values for further study of the IDZ model as well as to find the change regions in which the mass transfer rate maybe change suddenly.

\section{Acknowledgments}

This work is supported by the National Science Foundation of China (Contract no. 10732020 and 20476010), and the Natural Science Foundation of Beijing (Contract no. 1082002).

\section{References}

[1] V. V. Tarasov and D. X. Zhang, "Interfacial layer in nonequilibrium liquid-liquid systems," Doklady Physical Chemistry, vol. 350, no. 4-6, p. 271, 1996.

[2] D. X. Zhang, T. Shiyu, X. Rongshu, and V. V. Tarasov, “Mass transfer characteristic of interfacial layer activated by periodic mechanism in liquid-liquid system," Journal of Chemical Industry and Engineering, vol. 51, no. 1, pp. 108-114, 2000 (Chinese).

[3] D. Zhang and V. V. Tarasov, "Interfacial phenomena in liquid-liquid systems: new explanation of some peculiarities of mass transfer with chemical reactions," Journal of Chemical Industry and Engineering, vol. 52, no. 8, pp. 701-707, 2001 (Chinese).

[4] D. X. Zhang and V. V. Tarasov, "Electrostatic field effects on mass exchange of oxalic acid and copper di-2-ethylhexyl phosphate," Russian Journal of Physical Chemistry A, vol. 82, no. 3, pp. 506-511, 2008.

[5] D. X. Zhang, A.-M. Li, X. Xu, and V. V. Tarasov, "Effect of surfactants and static electric field on characteristics of liquid-liquid mass transfer kinetics," Transaction of Beijing Institute of Technology, vol. 28, no. 3, pp. 267-270, 2008.

[6] V. A. Vasilenko, E. M. Kol'tsova, V. V. Tarasov, Z. D. Xiang, and L. S. Gordeev, "Methods of nonequilibrium thermodynamics for studying and simulating mass transfer processes in liquidliquid systems," Theoretical Foundations of Chemical Engineering, vol. 41, no. 5, pp. 500-505, 2007. 
[7] D. X. Zhang and E. M. Koltsova, "Non-linear dynamics of mass transfer process with an interphase fractal structure in liquid-liquid system," Journal of Physics: Conference Series, vol. 96, no. 1, Article ID 012187, 8 pages, 2008.

[8] D. X. Zhang, Y. Z. Liao, Y. Tu, and H. S. Li, "Preparation of ultra fine copper oxide powder using precipitation on the interfaces of microemulsions," Transaction of Beijing Institute of Technology, vol. 25, no. 5, pp. 466-470, 2005.

[9] J. Li, S. F. Miao, and W. Zhang, "Analysis on bifurcations of multiple limit cycles for a parametrically and externally excited mechanical system," Chaos, Solitons E Fractals, vol. 31, no. 4, pp. 960-976, 2007.

[10] J. Li, Y. Tian, W. Zhang, and S. F. Miao, "Bifurcation of multiple limit cycles for a rotor-active magnetic bearings system with time-varying stiffness," International Journal of Bifurcation and Chaos, vol. 18, no. 3, pp. 755-778, 2008.

[11] V. V. Kapharov, Fundamentals of Mass Transfer, High School, Moscow, Russia, 1972.

[12] Z. D. Zhang and Q. S. Bi, "Bifurcation analysis of traveling wave solutions of a generalized CamassaHolm equation," in Proceedings of the 7th Conference on Nonlinear Dynamics, Nanjing, China, October 2004. 


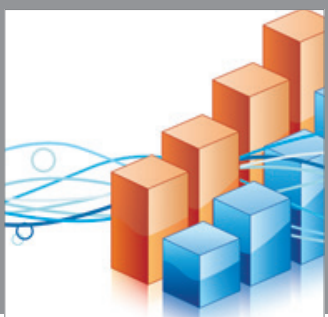

Advances in

Operations Research

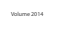

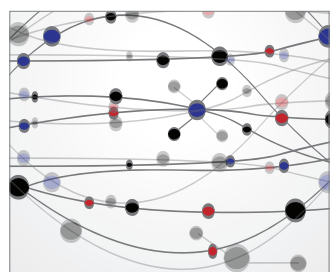

\section{The Scientific} World Journal
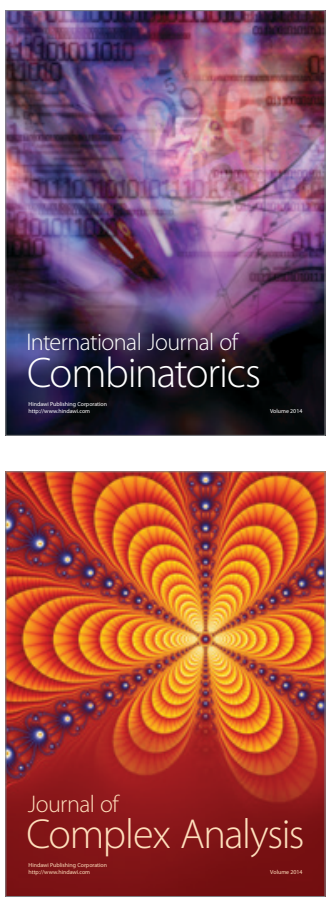

International Journal of

Mathematics and

Mathematical

Sciences
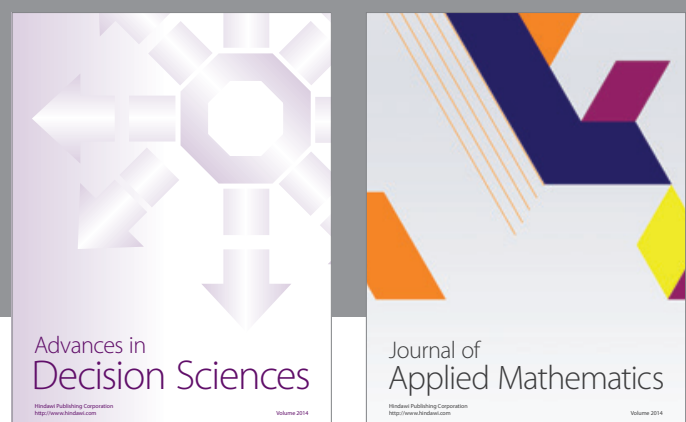

Journal of

Applied Mathematics
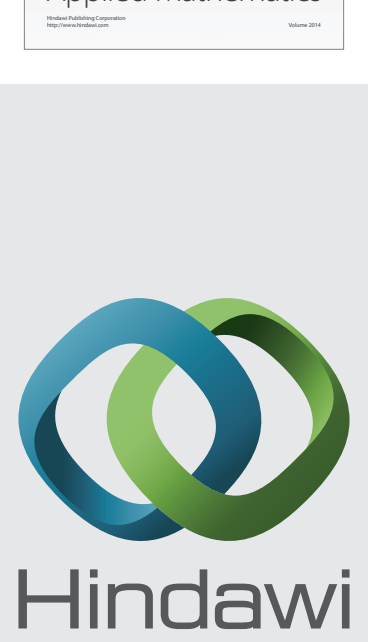

Submit your manuscripts at http://www.hindawi.com
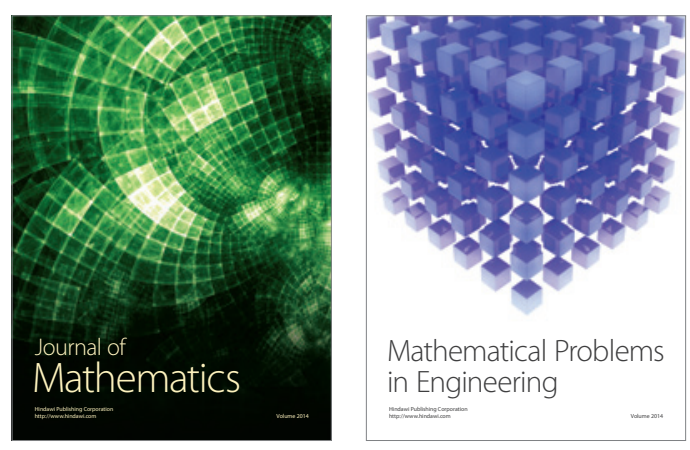

Mathematical Problems in Engineering
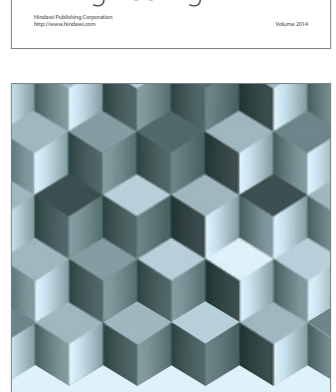

Journal of

Function Spaces
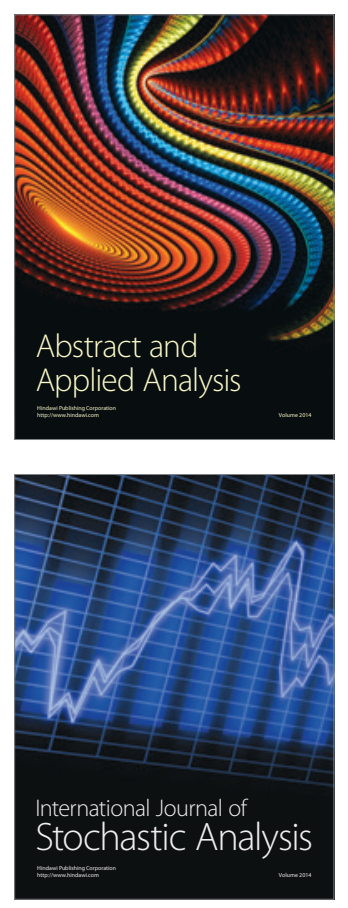

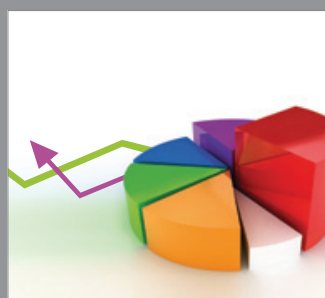

ournal of

Probability and Statistics

Promensencen
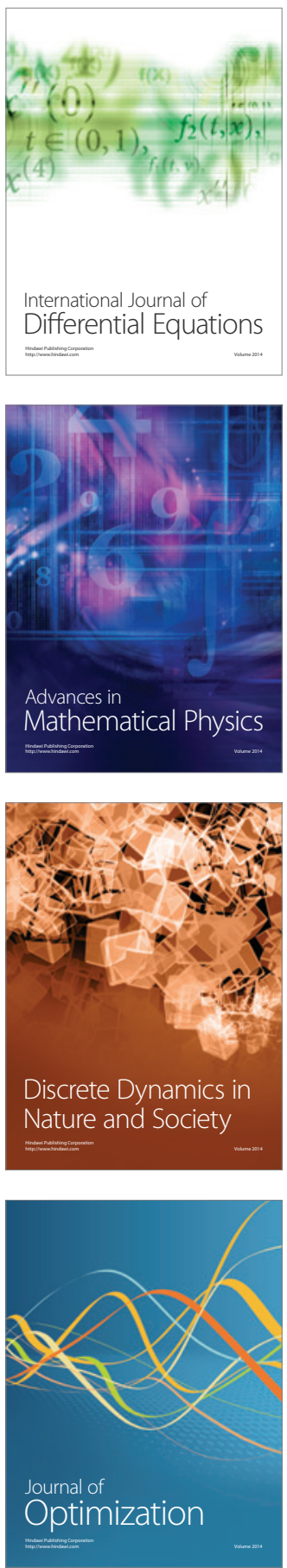